Preprint typeset in JHEP style - HYPER VERSION

hep-th/0703003

\title{
Current Algebra and Integrability of Principal Chiral Model on the World-sheet with General Metric
}

\author{
J. Klusoň * \\ Dipartimento di Fisica 83 Sezione I.N.F.N. \\ Università di Roma "Tor Vergata" \\ Via della Ricerca Scientifica 100133 Roma ITALY \\ E-mail: Josef.Kluson@roma2.infn.it
}

\begin{abstract}
We study the classical current algebra for principal chiral model defined on two dimensional world-sheet with general metric. We develop the Hamiltonian formalism and determine the form of the Poisson brackets between currents. Then we determine the Poisson bracket for Lax connection and we show that this Possion bracket does not depend on the world-sheet metric. We also study the Nambu-Gotto form of this model. We prove an existence of the Lax connection and determine their Poisson bracket.
\end{abstract}

KEYwOrds: Principal chiral model, integrability.

\footnotetext{
${ }^{*}$ On leave from Masaryk University, Brno
} 


\section{Contents}

1. Introduction 1

2. Hamiltonian formalism for principal chiral model 3

3. Current algebra 6

3.1 Comments on the gauge fixing 10

4. Nambu-Gotto form of principal model 12

5. Conclusion 15

\section{Introduction}

The discovery of the integrability of the classical string sigma model on $A d S_{5} \times S^{5}$ was one of the great achievements in the last few years [1] ${ }^{1}$. More precisely, the authors [1] found a Lax formulation of the equations of motion that leads to the existence of an infinite tower of conserved charges in the classical world-sheet theory. Using these charges it was possible to construct and classify large families of exact solutions of the classical equations of motion. However as was recently stressed in a very nice paper [四] this fact does not quite coincide with the standard definition of integrability. Integrability in the standard sense requires not only the existence of a tower of conserved charges but also requires that these charges be in involution. In other words the conserved charges should Poisson commute with each other. The knowledge of the Poisson brackets is necessary for constructing the action-angle variables for the system that plays an important role in semi-classical quantisation. The careful analysis presented in [4] explicitly demonstrated that for classical string moving on $R \times S^{3}$ submanifold of $A d S_{5} \times S^{5}$ that the Poisson brackets of conserved charges are in involution.

However it should be stressed that even if the bosonic string on $A d S_{5} \times S^{5}$ admits Lax formulation, there is a well known problem in determining the Poisson brackets of conserved charges. The problem is due to the presence of Non-Ultra Local terms in the Poisson brackets of the world-sheet fields that lead to the ambiguities in brackets for the charges. Some proposals for resolution of this issue were recently discussed in [13, 4]. For example, it was shown in 4 that there exists the resolution of this problem using the prescription of for regularising of the the problematic brackets that was introduced in the earlier work by Maillet [26, 27]. Explicitly, it was demonstrated in [4] that the application

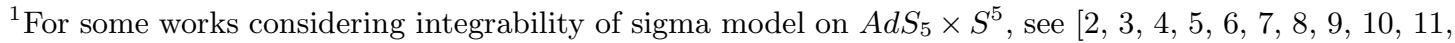
$12,13,14,15,16,17,18,19,20,21$
} 
of this procedure for the bosonic string in the conformal gauge moving on an $R \times S^{3}$ submanifold of the full $A d S_{5} \times S^{5}$ leads to the natural symplectic structure on the space of finite-gap solutions of the equations of motion that were constructed in [28].

As the next step it is natural to ask the question whether the principal chiral model is integrable on the world-sheet with general metric. An importance of the fact that one can define the Lax connection on the world-sheet with general metric was stressed in [14]. On the other hand the form of the current algebra for general metric is not known. Consequently it is also not known the form of the Poisson bracket of the Lax connection and hence it is not completely clear whether one can define infinite number of conserved charges that are in involution on the general world-sheet as well.

The goal of this paper is to answer these questions. We explicitly calculate the Poisson bracket of the principal chiral model on the world-sheet with general two dimensional metric. Even if the resulting Poisson bracket are complicated we will see that the Poisson bracket of the Lax connection does not depend on the metric at all and takes precisely the same form as was recently reviewed in [4]. According to the arguments given there it follows that the principal chiral model defined on the world-sheet with the general metric possesses again infinite number or conserved charges that are in involution.

It is important to stress that this result holds for the principal chiral model where the gauge symmetries are not fixed at all. The situation becomes more involved in case when the gauge fixing functions depend on the phase space variables. An example of such a more complicated gauge is uniform light cone [29, 30 ${ }^{2}$. The consistency of this gauge fixing implies that the Lagrange multipliers are completely fixed, the Virasoro constraints together with the gauge fixing conditions become the second class constraints and finally the original Hamiltonian strongly vanishes. In order to appropriate study this theory we should identify the Hamiltonian on the reduced phase space and also determine the Dirac brackets for the phase space variables. We leave the study of integrability and the current algebra for this gauge fixing theories for future.

We also discuss the Nambu-Gotto form of the principal chiral model. We demonstrate that it is again integrable and we will calculate the algebra of the currents. We also calculate the Poisson bracket of the Lax connection and we show that it takes the same form as in the paper 4 . This result again demonstrates an integrability of this form of the principal chiral model.

The organisation of the paper is as follows. In the next section (2) we introduce the basic notation of the principal chiral model defined on two dimensional world-sheet with general metric. In section (3) we calculate the algebra of currents. We also calculate the Poisson bracket of spatial component of the Lax connection. In section (团) we perform the Hamiltonian analysis of the Nambu-Gotto form of the principal chiral model. We show that this model is again integrable and we determine the algebra of current and also the Poisson bracket of the Lax connection for this model. In conclusion (5) we outline our results and suggest further directions of research.

\footnotetext{
${ }^{2}$ For recent discussion of this gauge, see for example [31, 32].
} 


\section{Hamiltonian formalism for principal chiral model}

In this section we introduce the Hamiltonian formalism for the principal chiral model defined on two-dimensional world-sheet with general metric $\gamma$. The action for this model takes the form

$$
\begin{aligned}
S & =-\frac{\sqrt{\lambda}}{4 \pi} \int d \tau d \sigma \sqrt{-\gamma} \gamma^{\alpha \beta} J_{\alpha}^{A} J_{\beta}^{B} K_{A B}= \\
& =-\frac{\sqrt{\lambda}}{4 \pi} \int d \tau d \sigma \sqrt{-\gamma} \gamma^{\alpha \beta} E_{M}^{A} \partial_{\alpha} x^{M} E_{N}^{B} \partial_{\beta} x^{N} K_{A B}= \\
& =-\frac{\sqrt{\lambda}}{4 \pi} \int d \tau d \sigma \sqrt{-\gamma} \gamma^{\alpha \beta} G_{M N} \partial_{\alpha} x^{M} \partial_{\beta} x^{N} .
\end{aligned}
$$

Here $\frac{\sqrt{\lambda}}{2 \pi}$ is the effective string tension. Coordinates $\sigma$ and $\tau$ parametrise the string worldsheet. We assume the range of $\sigma$ to be $-\pi \leq \sigma \leq \pi$. The current $J_{\alpha}^{A}$ is defined through the group element $g$ that belongs to the group $G$ as

$$
J_{\alpha}=g^{-1} \partial_{\alpha} g \equiv J_{\alpha}^{A} T_{A}
$$

where $T_{A}$ form the basis of the algebra $\mathrm{g}$ that obey the relations

$$
\operatorname{Tr}\left(T_{A} T_{B}\right)=K_{A B}, \quad\left[T_{A}, T_{B}\right]=f_{A B}^{C} T_{C},
$$

where $K_{A B}$ is invertible matrix and where $f_{A B}^{C}=-f_{B A}^{C}$ are structure constants of the algebra $\mathbf{g}$. The indices $A, B$ label components of the basis $T_{A}$. If we parametrise the group element with the fields $x^{M}$ we can write the current $J_{\alpha}^{A}$ as

$$
J_{\alpha}^{A}=E_{M}^{A} \partial_{\alpha} x^{M} .
$$

Finally we have introduced the metric

$$
G_{M N}=E_{M}^{A} K_{A B} E_{N}^{B}
$$

defined on some target manifold labelled with coordinates $x^{M}$. In this interpretation $E_{M}^{A}$ are vielbeins of the target manifold [33].

We now develop the Hamiltonian formalism for this principal chiral model, where we consider $x^{M}$ and corresponding momenta $p_{M}$ as canonical variables. There also exists an alternative form of the Hamiltonian formalism based on presumption that the current $J_{\sigma}^{A}$ is the dynamical variable [22] (For some recent works, see for example [13, 25, 2]). It can be shown that these two approaches give equivalent result.

The conjugate momenta to $x^{M}$ are equal to

$$
p_{M}=-\frac{\sqrt{\lambda}}{2 \pi} \sqrt{-\gamma} \gamma^{\tau \alpha} E_{M}^{A} K_{A B} J_{\alpha}^{B}
$$

and together with $x^{M}$ obey the canonical Poisson brackets

$$
\left\{x^{M}(\sigma), p_{N}\left(\sigma^{\prime}\right)\right\}=\delta_{N}^{M} \delta\left(\sigma-\sigma^{\prime}\right) .
$$


Then the Hamiltonian takes the form

$$
\begin{aligned}
H_{0} & =\int_{0}^{2 \pi} d \sigma \mathcal{H}_{0}=\int_{0}^{2 \pi} d \sigma\left[\partial_{\tau} x^{M} p_{M}-\mathcal{L}\right]= \\
& =\int_{0}^{2 \pi} d \sigma\left[-\frac{1}{\sqrt{-\gamma} \gamma^{\tau \tau}}\left(\frac{\pi}{\sqrt{\lambda}} G^{M N} p_{M} p_{N}+\frac{\sqrt{\lambda}}{4 \pi} G_{M N} \partial_{\sigma} x^{M} \partial_{\sigma} x^{N}\right)-\frac{\gamma^{\tau \sigma}}{\gamma^{\tau \tau}} p_{M} \partial_{\sigma} x^{M}\right] .
\end{aligned}
$$

Note that the action is invariant under Weyl transformation

$$
\gamma_{\alpha \beta}^{\prime}(\sigma, \tau)=e^{\phi(\sigma, \tau)} \gamma_{\alpha \beta}(\sigma, \tau)
$$

In what follows we use following parametrisation of the metric variables $\gamma_{\alpha \beta}$

$$
\lambda^{ \pm}=\frac{\sqrt{-\gamma} \pm \gamma_{\tau \sigma}}{\gamma_{\sigma \sigma}}, \quad \xi=\ln \gamma_{\sigma \sigma}
$$

where $\lambda^{ \pm}$are manifestly invariant under (2.9) while $\xi$ transforms as $\xi^{\prime}(\sigma, \tau)=\xi(\sigma, \tau)+$ $\phi(\sigma, \tau)$. Then the Hamiltonian density $\mathcal{H}_{0}$ is equal to

$$
\begin{aligned}
& \mathcal{H}_{0}=\frac{\lambda^{+}+\lambda^{-}}{2} T_{0}+\frac{\lambda^{+}-\lambda^{-}}{2} T_{1}=\lambda^{+} T_{+}+\lambda^{-} T_{-}, \\
& T_{+}=\frac{1}{2}\left(T_{0}+T_{1}\right), \quad T_{-}=\frac{1}{2}\left(T_{0}-T_{1}\right),
\end{aligned}
$$

where

$$
\begin{aligned}
& T_{1} \equiv p_{M} \partial_{\sigma} x^{M}, \\
& T_{0} \equiv \frac{\pi}{\sqrt{\lambda}} p_{M} G^{M N} p_{N}+\frac{\sqrt{\lambda}}{4 \pi} \partial_{\sigma} x^{M} G_{M N} \partial_{\sigma} x^{N} .
\end{aligned}
$$

Since the action (2.1) does not contain time-derivative of $\gamma_{\alpha \beta}$ it follows that the momenta conjugate to $\lambda^{ \pm}, \xi$ are zero:

$$
\pi^{ \pm}=\frac{\delta S}{\delta \partial_{\tau} \lambda^{ \pm}}=0, \quad \pi^{\xi}=\frac{\delta S}{\delta \partial_{\tau} \xi}=0
$$

These conditions are primary constraints of the theory. According to the general theory of the constraint systems [34, 35, 36] the primary constraints should be preserved during the time evolution of the system

$$
\dot{\pi}_{ \pm}=\left\{\pi_{ \pm}, H_{0}\right\}=T_{ \pm} \approx 0
$$

Then the consistency of the theory implies two secondary constraints

$$
T_{+}=T_{-}=0
$$


Now we have to calculate the Poisson brackets between constraints $T_{1}(\sigma), T_{0}\left(\sigma^{\prime}\right)$ and also between $p_{N}, x^{M}$ and $T_{0}, T_{1}$.

$$
\begin{aligned}
\left\{p_{N}(\sigma), T_{1}\left(\sigma^{\prime}\right)\right\} & =-p_{N}\left(\sigma^{\prime}\right) \partial_{\sigma^{\prime}} \delta\left(\sigma-\sigma^{\prime}\right) \\
\left\{p_{N}(\sigma), T_{0}\left(\sigma^{\prime}\right)\right\} & =-\frac{\pi}{\sqrt{\lambda}} p_{K}\left(\sigma^{\prime}\right) \partial_{N} G^{K L} p_{L}\left(\sigma^{\prime}\right) \delta\left(\sigma-\sigma^{\prime}\right)- \\
& -\frac{\sqrt{\lambda}}{2 \pi} \partial_{\sigma^{\prime}} \delta\left(\sigma-\sigma^{\prime}\right) G_{N K}\left(\sigma^{\prime}\right) \partial_{\sigma^{\prime}} x^{K}\left(\sigma^{\prime}\right)- \\
& -\frac{\sqrt{\lambda}}{4 \pi} \partial_{\sigma^{\prime}} x^{K}\left(\sigma^{\prime}\right) \partial_{N} G_{K L} \partial_{\sigma^{\prime}} x^{L}\left(\sigma^{\prime}\right) \delta\left(\sigma-\sigma^{\prime}\right), \\
\left\{x^{N}(\sigma), T_{1}\left(\sigma^{\prime}\right)\right\} & =\partial_{\sigma^{\prime}} x^{N}\left(\sigma^{\prime}\right) \delta\left(\sigma-\sigma^{\prime}\right) \\
\left\{x^{N}(\sigma), T_{0}\left(\sigma^{\prime}\right)\right\} & =\frac{2 \pi}{\sqrt{\lambda}} G^{N L} p_{L}\left(\sigma^{\prime}\right) \delta\left(\sigma-\sigma^{\prime}\right) .
\end{aligned}
$$

Then it is easy to determine Poisson brackets between $T_{1}, T_{0}$

$$
\begin{aligned}
& \left\{T_{1}(\sigma), T_{1}\left(\sigma^{\prime}\right)\right\}=\partial_{\sigma} T_{1}(\sigma) \delta\left(\sigma-\sigma^{\prime}\right)+2 T_{1}(\sigma) \partial_{\sigma} \delta\left(\sigma-\sigma^{\prime}\right), \\
& \left\{T_{1}(\sigma), T_{0}\left(\sigma^{\prime}\right)\right\}=\partial_{\sigma} T_{0}(\sigma) \delta\left(\sigma-\sigma^{\prime}\right)+2 T_{0}(\sigma) \partial_{\sigma} \delta\left(\sigma-\sigma^{\prime}\right), \\
& \left\{T_{0}(\sigma), T_{0}\left(\sigma^{\prime}\right)\right\}=\partial_{\sigma} T_{1}(\sigma) \delta\left(\sigma-\sigma^{\prime}\right)+2 T_{1}(\sigma) \partial_{\sigma} \delta\left(\sigma-\sigma^{\prime}\right) .
\end{aligned}
$$

Using these Poisson brackets we finally get

$$
\begin{aligned}
& \left\{T_{+}(\sigma), T_{+}\left(\sigma^{\prime}\right)\right\}=\partial_{\sigma} T_{+}(\sigma) \delta\left(\sigma-\sigma^{\prime}\right)+2 T_{+}(\sigma) \partial_{\sigma} \delta\left(\sigma-\sigma^{\prime}\right), \\
& \left\{T_{-}(\sigma), T_{-}\left(\sigma^{\prime}\right)\right\}=-\partial_{\sigma} T_{-}(\sigma) \delta\left(\sigma-\sigma^{\prime}\right)-2 T_{-}(\sigma) \partial_{\sigma} \delta\left(\sigma-\sigma^{\prime}\right), \\
& \left\{T_{+}(\sigma), T_{-}\left(\sigma^{\prime}\right)\right\}=0
\end{aligned}
$$

These results imply that the constraints $T_{0}, T_{1}$ do not generate any additional ones. We denote all constraints as $\Phi_{\alpha}$, where $\alpha=\left(\lambda^{ \pm}, \xi, \pm\right)$ label the first class constraints $\pi_{ \pm}^{\lambda}=$ $\pi_{\xi}=0$ and $T_{ \pm}=0$. We introduce the Lagrange multipliers $\rho^{\alpha}$. Then the generalised Hamiltonian density takes the form

$$
\mathcal{H}_{T}=\mathcal{H}_{0}+\rho^{\alpha}(p, x) \Phi_{\alpha},
$$

where in principle $\rho^{\alpha}$ depend on the phase space variables $p_{M}, x^{M}, \pi_{ \pm}^{\lambda}, \lambda^{ \pm}, \pi_{\xi}, \xi$. Then (2.19) implies that the equation of motion for any $F$ defined on the phase space takes the form

$$
\begin{aligned}
\frac{d F}{d \tau} & =\partial_{\tau} F+\left\{F, H_{T}\right\}= \\
& =\partial_{\tau} F+\left\{F, H_{0}\right\}+\int d \sigma\left(\rho^{\alpha}(\sigma)\left\{F, \Phi_{\alpha}(\sigma)\right\}+\left\{F, \rho^{\alpha}(\sigma)\right\} \Phi_{\alpha}(\sigma)\right) \approx \\
& \approx \partial_{\tau} F+\left\{F, H_{0}\right\}+\int d \sigma \rho^{\alpha}(\sigma)\left\{F, \Phi_{\alpha}(\sigma)\right\},
\end{aligned}
$$

where $\approx$ means that this equation holds on the constraint surface $\Phi_{\alpha} \approx 0$. 


\section{Current algebra}

In this section we will calculate the current algebra of the principal chiral model defined on the world-sheet with general metric. Recall that these currents are defined as

$$
J_{\alpha}^{A}=E_{M}^{A} \partial_{\alpha} x^{M} .
$$

In order to calculate the current algebra we have to express these currents using the canonical variables $x^{M}, p_{M}$. Namely we have to find the dependence $\partial_{\tau} x^{M}$ on $p_{M}, x^{M}$. Using the Hamiltonian (2.19) we find the time derivative of $x^{M}$ as

$$
\begin{aligned}
\partial_{\tau} x^{M}(\sigma) & =\left\{x^{M}(\sigma), H_{T}\right\}=\frac{\left(\lambda^{+}+\lambda^{-}+\rho^{+}+\rho^{-}\right)}{2} \frac{2 \pi}{\sqrt{\lambda}} G^{M N} p_{N}+\frac{\lambda^{+}-\lambda^{-}+\rho^{+}-\rho^{-}}{2} \partial_{\sigma} x^{M}= \\
& =\left(\frac{\sqrt{-\gamma}}{\gamma_{\sigma \sigma}}+\rho^{0}\right) \frac{2 \pi}{\sqrt{\lambda}} G^{M N} p_{N}+\left(\frac{\gamma_{\tau \sigma}}{\gamma_{\sigma \sigma}}+\rho^{1}\right) \partial_{\sigma} x^{M}
\end{aligned}
$$

and hence

$$
J_{\tau}^{A}=\frac{2 \pi}{\sqrt{\lambda}}\left(-\frac{1}{\sqrt{-\gamma} \gamma^{\tau \tau}}+\rho^{0}\right) K^{A B} E_{B}^{M} p_{M}+\left(-\frac{\gamma^{\tau \sigma}}{\gamma^{\tau \tau}}+\rho^{1}\right) E_{M}^{A} \partial_{\sigma} x^{M}
$$

where

$$
E_{B}^{M} E_{M}^{C}=\delta_{B}^{C}, \quad E_{M}^{B} E_{B}^{N}=\delta_{M}^{N} .
$$

Our goal is to calculate the algebra of currents defined above. To do this we have to make presumptions about the dependence of the Lagrange multipliers $\rho^{ \pm}$on the phase space variables. Since we work in the theory where the gauge symmetry is not fixed it is natural to presume that the Lagrange multipliers $\rho^{ \pm}$depend on $\sigma, \tau$ only [36]: $\rho^{ \pm}=\rho(t, \sigma)$. Then it turns out that it is useful to define

$$
-\frac{1}{\sqrt{-\tilde{\gamma}} \tilde{\gamma}^{\tau \tau}}=-\frac{1}{\sqrt{-\gamma} \gamma^{\tau \tau}}+\rho^{0}, \quad-\frac{\tilde{\gamma}^{\tau \sigma}}{\tilde{\gamma}^{\tau \tau}}=-\frac{\gamma^{\tau \sigma}}{\gamma^{\tau \tau}}+\rho^{1}
$$

so that we can write

$$
J_{\tau}^{A}=-\frac{2 \pi}{\sqrt{\lambda}} \frac{1}{\sqrt{-\tilde{\gamma}} \tilde{\gamma}^{\tau \tau}} K^{A B} E_{B}^{M} p_{M}-\frac{\tilde{\gamma}^{\tau \sigma}}{\tilde{\gamma}^{\tau \tau}} E_{M}^{A} \partial_{\sigma} x^{M}
$$

In the same way we also get

$$
\mathcal{H}_{T}=-\frac{1}{\sqrt{-\tilde{\gamma}} \tilde{\gamma}^{\tau \tau}} T_{0}-\frac{\tilde{\gamma}^{\tau \sigma}}{\tilde{\gamma}^{\tau \tau}} T_{1}+\rho_{\lambda}^{+} \pi_{+}^{\lambda}+\rho_{\lambda}^{-} \pi_{-}^{\lambda}+\rho^{\xi} \lambda_{-} .
$$

Then the generalised action takes the form

$$
\begin{aligned}
S & =\int d \sigma d \tau\left[\partial_{\tau} x^{M} p_{M}+\partial_{\tau} \lambda^{+} \pi_{+}+\partial_{\tau} \lambda^{-} \pi_{-}+\partial_{\tau} \xi \pi_{\xi}-\mathcal{H}_{T}\right]= \\
& =-\frac{\sqrt{\lambda}}{4 \pi} \int d \sigma d \tau \sqrt{-\tilde{\gamma}} \tilde{\gamma}^{\alpha \beta} J_{\alpha}^{A} J_{\beta}^{B} K_{A B}+ \\
& +\int d \sigma d \tau\left(\partial_{\tau} \lambda^{+} \pi_{+}^{\lambda}+\partial_{\tau} \lambda^{-} \pi_{-}^{\lambda}+\partial_{\tau} \lambda^{\xi} \pi_{\xi}-\rho_{\lambda}^{+} \pi_{+}^{\lambda}-\rho_{\lambda}^{-} \pi_{-}^{\lambda}-\rho^{\xi} \pi_{\xi}\right) .
\end{aligned}
$$


Then from (3.8) we determine the equations of motion for current $J_{\alpha}$ in the form

$$
\partial_{\alpha}\left[\sqrt{-\tilde{\gamma}} \tilde{\gamma}^{\alpha \beta} J_{\beta}^{A}\right]=0
$$

Let us now introduce the Lax connection for given principal model. We define its components as

$$
\hat{J}_{\alpha}(\Lambda)=\frac{1}{1-\Lambda^{2}}\left(J_{\alpha}-\Lambda \tilde{\gamma}_{\alpha \beta} \epsilon^{\beta \gamma} J_{\gamma}\right)
$$

where

$$
\epsilon^{\alpha \beta}=\frac{e^{\alpha \beta}}{\sqrt{-\tilde{\gamma}}}, \quad e^{\alpha \beta}=-e^{\beta \alpha}, \quad e^{01}=1
$$

and where $\Lambda$ is a spectral parameter. Note that the Lax connection depends on $\tilde{\gamma}$ that is combination of the world-sheet metric $\gamma$ and the gauge parameters $\rho^{ \pm}$. Then using the equations of motion (3.9) and also using the fact that $J_{\alpha}^{A}$ obey the flatness condition

$$
\partial_{\alpha} J_{\beta}^{A}-\partial_{\beta} J_{\alpha}^{A}+J_{\alpha}^{B} J_{\beta}^{C} f_{B C}^{A}=0
$$

that follows from the definition of the currents $J_{\alpha}^{A}$ given in (2.2) we can show that $\hat{J}$ is flat

$$
\partial_{\alpha} \hat{J}_{\beta}^{A}-\partial_{\beta} \hat{J}_{\alpha}^{A}+\hat{J}_{\alpha}^{B} \hat{J}_{\beta}^{C} f_{B C}^{A}=0
$$

Now we are going to calculate the Poisson brackets between currents $J_{\alpha}$. Firstly, it is clear that

$$
\left\{J_{\sigma}^{A}(\sigma), J_{\sigma}^{B}\left(\sigma^{\prime}\right)\right\}=0 .
$$

On the other hand explicit calculation gives

$$
\begin{aligned}
& \left\{J_{\tau}^{A}(\sigma), J_{\sigma}^{B}\left(\sigma^{\prime}\right)\right\}= \\
= & -\frac{2 \pi}{\sqrt{\lambda}} \frac{1}{\sqrt{-\tilde{\gamma}} \tilde{\gamma}^{\tau \tau}(\sigma)} K^{A B} \partial_{\sigma} \delta\left(\sigma-\sigma^{\prime}\right)-\frac{2 \pi}{\sqrt{\lambda}} \frac{1}{\sqrt{-\tilde{\gamma}} \tilde{\gamma}^{\tau \tau}(\sigma)} K^{A C} E_{C}^{M}(\sigma) \partial_{N} E_{M}^{B}(\sigma) \partial_{\sigma} x^{N}(\sigma) \delta\left(\sigma-\sigma^{\prime}\right)+ \\
+ & \frac{2 \pi}{\sqrt{\lambda}} \frac{1}{\sqrt{-\tilde{\gamma}} \tilde{\gamma}^{\tau \tau}(\sigma)} K^{A C} E_{C}^{M}(\sigma) \partial_{M} E_{N}^{B}(\sigma) \partial_{\sigma} x^{N}(\sigma) \delta\left(\sigma-\sigma^{\prime}\right)= \\
= & -\frac{2 \pi}{\sqrt{\lambda}} \frac{1}{\sqrt{-\tilde{\gamma}} \tilde{\gamma}^{\tau \tau}(\sigma)} K^{A B} \partial_{\sigma} \delta\left(\sigma-\sigma^{\prime}\right)-\frac{2 \pi}{\sqrt{\lambda}} \frac{1}{\sqrt{-\tilde{\gamma}} \tilde{\gamma}^{\tau \tau}(\sigma)} J_{\sigma}^{C} f_{C D}^{A} K^{D B} \delta\left(\sigma-\sigma^{\prime}\right),
\end{aligned}
$$

where we have used

$$
f\left(\sigma^{\prime}\right) \partial_{\sigma} \delta\left(\sigma-\sigma^{\prime}\right)=f(\sigma) \partial_{\sigma} \delta\left(\sigma-\sigma^{\prime}\right)+\partial_{\sigma} f(\sigma) \delta\left(\sigma-\sigma^{\prime}\right)
$$

and also the flatness condition of the current $J_{\alpha}^{A}(3.12)$ that implies

$$
\partial_{N} E_{M}^{A}-\partial_{M} E_{N}^{A}+E_{N}^{B} E_{M}^{C} f_{B C}^{A}=0 .
$$


For letter purposes we also determine the Poisson brackets between $J_{\tau}$ and $p_{M}, x^{M}$

$$
\begin{aligned}
\left\{J_{\tau}^{A}(\sigma), p_{M}\left(\sigma^{\prime}\right)\right\} & =-\frac{2 \pi}{\sqrt{\lambda}} \frac{1}{\sqrt{-\tilde{\gamma}} \tilde{\gamma}^{\tau \tau}(\sigma)} K^{A B} \partial_{M} E_{B}^{N} p_{N}(\sigma)- \\
& -\frac{\tilde{\gamma}^{\tau \sigma}(\sigma)}{\tilde{\gamma}^{\tau \tau}(\sigma)} \partial_{M} E_{N}^{A} \partial_{\sigma} x^{N} \delta\left(\sigma-\sigma^{\prime}\right)-E_{M}^{A} \frac{\tilde{\gamma}^{\tau \sigma}(\sigma)}{\tilde{\gamma}^{\tau \tau}(\sigma)} \partial_{\sigma} \delta\left(\sigma-\sigma^{\prime}\right), \\
\left\{J_{\sigma}^{A}(\sigma), p_{M}\left(\sigma^{\prime}\right)\right\} & =\partial_{M} E_{N}^{N} \partial_{\sigma} x^{N}(\sigma) \delta\left(\sigma-\sigma^{\prime}\right)+E_{M}^{A} \partial_{\sigma} \delta\left(\sigma-\sigma^{\prime}\right)
\end{aligned}
$$

and

$$
\left\{J_{\tau}^{A}(\sigma), x^{M}\left(\sigma^{\prime}\right)\right\}=\frac{2 \pi}{\sqrt{\lambda}} \frac{1}{\sqrt{-\tilde{\gamma}} \tilde{\gamma}^{\tau \tau}(\sigma)} K^{A B} E_{B}^{M} \delta\left(\sigma-\sigma^{\prime}\right) .
$$

Then we obtain

$$
\begin{aligned}
& \left\{J_{\tau}^{A}(\sigma), J_{\tau}^{B}\left(\sigma^{\prime}\right)\right\}=-\frac{2 \pi}{\sqrt{\lambda}}\left\{J_{\tau}^{A}(\sigma), E_{D}^{M}\left(\sigma^{\prime}\right)\right\} K^{B D} p_{M}\left(\sigma^{\prime}\right) \frac{1}{\sqrt{-\tilde{\gamma}} \tilde{\gamma}^{\tau \tau}\left(\sigma^{\prime}\right)}- \\
- & \left\{J_{\tau}^{A}(\sigma), J_{\sigma}^{B}\left(\sigma^{\prime}\right)\right\} \frac{\tilde{\gamma}^{\tau \sigma}\left(\sigma^{\prime}\right)}{\tilde{\gamma}^{\tau \tau}\left(\sigma^{\prime}\right)}-\frac{2 \pi}{\sqrt{\lambda}}\left\{J_{\tau}^{A}(\sigma), p_{M}\left(\sigma^{\prime}\right)\right\} K^{B D} E_{D}^{M}\left(\sigma^{\prime}\right) \frac{1}{\sqrt{-\tilde{\gamma}} \tilde{\gamma}^{\tau \tau}\left(\sigma^{\prime}\right)}= \\
= & -\frac{2 \pi}{\sqrt{\lambda}} \frac{1}{\sqrt{-\tilde{\gamma}}(\sigma) \tilde{\gamma}^{\tau \tau}(\sigma)} J_{\tau}^{C}(\sigma) f_{C D}^{A} K^{D B} \delta\left(\sigma-\sigma^{\prime}\right)+ \\
+ & \frac{2 \pi}{\sqrt{\lambda}} \frac{\tilde{\gamma}^{\tau \sigma}(\sigma)}{\sqrt{-\tilde{\gamma}}(\sigma) \tilde{\gamma}^{\tau \tau}(\sigma)} J_{\sigma}^{C}(\sigma) f_{C D}^{A} K^{D B} \delta\left(\sigma-\sigma^{\prime}\right)+ \\
+ & \frac{2 \pi}{\sqrt{\lambda}}\left[\frac{1}{\sqrt{-\tilde{\gamma}}(\sigma) \tilde{\gamma}^{\tau \tau}(\sigma)} \frac{\tilde{\gamma}^{\sigma \tau}\left(\sigma^{\prime}\right)}{\tilde{\gamma}^{\tau \tau}\left(\sigma^{\prime}\right)}+\frac{1}{\sqrt{-\tilde{\gamma}}\left(\sigma^{\prime}\right) \tilde{\gamma}^{\tau \tau}\left(\sigma^{\prime}\right)} \frac{\tilde{\gamma}^{\tau \sigma}(\sigma)}{\tilde{\gamma}^{\tau \tau}(\sigma)}\right] K^{A B} \partial_{\sigma} \delta\left(\sigma-\sigma^{\prime}\right)
\end{aligned}
$$

using

$$
\begin{aligned}
& \partial_{M} E_{A}^{N}=-E_{A}^{P} \partial_{M} E_{P}^{B} E_{B}^{N} \\
& E_{C}^{M} \partial_{M} E_{D}^{N}-E_{D}^{M} \partial_{M} E_{C}^{N}=f_{C D}^{A} E_{A}^{N}
\end{aligned}
$$

With the help of the Poisson brackets (3.15) and (3.20) we can determine the Poisson bracket between the spatial components of the Lax connection for two different spectral parameters $\Lambda, \Gamma$

$$
\begin{aligned}
& \left\{\hat{J}_{\sigma}^{A}(\sigma, \Lambda), \hat{J}_{\sigma}^{B}\left(\sigma^{\prime}, \Gamma\right)\right\}= \\
= & -\frac{2 \pi}{\sqrt{\lambda}} \frac{K^{A B}(\Gamma+\Lambda)}{\left(1-\Lambda^{2}\right)\left(1-\Gamma^{2}\right)} \partial_{\sigma} \delta\left(\sigma-\sigma^{\prime}\right)-\frac{2 \pi}{\sqrt{\lambda}} \frac{(\Lambda+\Gamma)}{\left(1-\Lambda^{2}\right)\left(1-\Gamma^{2}\right)} J_{\sigma}^{C}(\sigma) f_{C D}^{A} K^{D B} \delta\left(\sigma-\sigma^{\prime}\right)- \\
- & \frac{2 \pi}{\sqrt{\lambda}} \frac{\Lambda \Gamma}{\left(1-\Lambda^{2}\right)\left(1-\Gamma^{2}\right)}\left(\sqrt{-\tilde{\gamma}} \tilde{\gamma}^{\tau \tau} J_{\tau}^{C}+\sqrt{-\tilde{\gamma}} \tilde{\gamma}^{\tau \sigma} J_{\sigma}^{C}\right)(\sigma) f_{C D}^{A} K^{D B} \delta\left(\sigma-\sigma^{\prime}\right) .
\end{aligned}
$$

The expression above can be rewritten into the form that on the right side contains the combinations of the spatial components of the Lax connection and term that is proportional 
to the non-ultra-local term $\partial_{\sigma} \delta\left(\sigma-\sigma^{\prime}\right)$

$$
\begin{gathered}
\frac{\sqrt{\lambda}}{2 \pi}\left\{\hat{J}_{\sigma}^{A}(\sigma, \Lambda), \hat{J}_{\sigma}^{B}\left(\sigma^{\prime}, \Gamma\right)\right\}=-\frac{K^{A B}(\Gamma+\Lambda)}{\left(1-\Lambda^{2}\right)\left(1-\Gamma^{2}\right)} \partial_{\sigma} \delta\left(\sigma-\sigma^{\prime}\right)- \\
-\frac{\Gamma^{2}}{\left(1-\Gamma^{2}\right)(\Lambda-\Gamma)} \hat{J}_{\sigma}^{C}(\Lambda) f_{C D}^{A} K^{D B} \delta\left(\sigma-\sigma^{\prime}\right)-\frac{\Lambda^{2}}{\left(1-\Lambda^{2}\right)(\Lambda-\Gamma)} \hat{J}_{\sigma}^{C}(\Gamma) f_{C D}^{A} K^{D B} \delta\left(\sigma-\sigma^{\prime}\right) .
\end{gathered}
$$

This result has precisely the same form (if we use the tensor notation) as the Poisson bracket given in 四. Since (3.23) does not explicitly depend on the world-sheet metric $\gamma_{\alpha \beta}$ and the gauge parameters $\rho^{ \pm}$we obtain that the Poisson bracket between spatial components of the Lax connections is gauge invariant. This result has important consequence for the integrability of the theory. Recall that the monodromy matrix is defined as $[22,23,24,26$, 27]

$$
\mathcal{T}(l, \Lambda)=P \exp \left(\int_{l} \hat{J}_{\alpha}(\Lambda) \frac{d x^{\alpha}}{d \xi}\right)
$$

where $l=\left(x^{\alpha}(\xi) \equiv\left(\tau^{\prime}(\xi), \sigma^{\prime}(\xi)\right)\right)$ is a curve on two dimensional cylinder, $\hat{J}_{\alpha} \frac{d x^{\alpha}}{d \xi}$ is an embedding of the Lax connection with spectral parameter $\Lambda$ on the curve $l$ and $\xi$ is a parameter that labels points on given curve. Finally $P$ in (3.24) means path ordering. We will calculate the Poisson bracket of the monodromy matrices that are defined at equal time $\tau$. In this case the curve $l$ is defined as

$$
l=\left(\sigma^{\prime}=\xi, \xi \in\left(x_{1}, y_{1}\right)\right)
$$

Then for the curve $(3.25)$ the monodromy matrix $\mathcal{T}$ takes the form

$$
\mathcal{T}\left(\Lambda, x_{1}, y_{1}\right)=P \exp \left(\int_{x_{1}}^{y_{1}} d \sigma^{\prime} \hat{J}_{\sigma}\left(\tau, \sigma^{\prime}\right)\right) .
$$

As was shown in [22, 23, 24, 26, 27] the Poisson brackets between elements of the monodromy matrices $\mathcal{T}\left(\Lambda, x_{1}, y_{1}\right)$ and $\mathcal{T}\left(\Gamma, x_{2}, y_{2}\right)$ are equal to

$$
\begin{array}{r}
\left\{\mathcal{T}_{\alpha \beta}\left(x_{1}, y_{1}, \Lambda\right), \mathcal{T}_{\gamma \delta}\left(x_{2}, y_{2}, \Gamma\right)\right\}=\int_{x_{1}}^{y_{1}} d \sigma \int_{x_{2}}^{y_{2}} d \sigma^{\prime} \mathcal{T}_{\alpha \omega_{1}}\left(x_{1}, \sigma, \Lambda\right) \mathcal{T}_{\omega_{2} \beta}\left(\sigma, y_{1}, \Lambda\right) \times \\
\times\left\{\hat{J}_{\omega_{1} \omega_{2}}(\sigma, \Lambda), \hat{J}_{\rho_{1} \rho_{2}}\left(\sigma^{\prime}, \Gamma\right)\right\} \mathcal{T}_{\gamma \rho_{1}}\left(x_{2}, \sigma^{\prime}, \Gamma\right) \mathcal{T}_{\rho_{2} \delta}\left(\sigma^{\prime}, y_{2}^{\prime}, \Gamma\right),
\end{array}
$$

where $\alpha, \beta, \ldots$ label the matrix indices of $\mathcal{T}$. The form of the Poisson bracket above shows a significance of the Poisson bracket (3.23) for the calculation of the Poisson brackets between transition matrices and then between the Poisson brackets of the conserved local charges. Recall that these local conserved charges arise from the expansion of the trace of the monodromy matrix with respect to the corresponding spectral parameter 粗. Then since (3.23) is gauge invariant and metric independent and takes completely the same form as in [4] we immediately obtain that we can find an infinite number of local conserved charges 
that are in involution even on the string world-sheet with general metric. Of course all these results depend on the presumption that the Lagrange multipliers $\rho^{ \pm}$do not depend on the phase space variables. In fact this is natural presumption in case when we do not fix the gauge since the gauge symmetries imply the appearance of arbitrary functions in the general solutions of the equations of motion. On the other hand if we fix the gauge then Lagrange multipliers can depend on the phase space variables. Let us discuss this issue in more detail.

\subsection{Comments on the gauge fixing}

We now present some comments considering the gauge fixing for the principal chiral model defined on world-sheet with general metric. Recall that there are five first class constraints defined above the equation (2.19)

$$
\Phi_{\alpha} \approx 0
$$

Fixing these constrains is achieved by introducing additional constraints [34, 35, 36]

$$
G_{\alpha}(\sigma) \approx 0
$$

that have nonzero Poisson bracket with $\Phi_{\alpha}$ on the constraint surface

$$
\left\{G_{\alpha}(\sigma), \Phi_{\beta}\left(\sigma^{\prime}\right)\right\}=C_{\alpha \beta}\left(\sigma, \sigma^{\prime}\right)
$$

where the matrix $C_{\alpha \beta}\left(\sigma, \sigma^{\prime}\right)$ is non-singular. Then the extended set of constraints $\Phi_{\alpha}, G_{\alpha}$

consists the second class constraints. In fact, the consistency of the theory demands that these constraints are preserved during the time-evolution of the system. Then the Hamiltonian equation of motion for $G_{\alpha}$ implies

$$
\begin{aligned}
\frac{d}{d \tau} G_{\alpha}(\sigma) & =\partial_{\tau} G_{\alpha}(\sigma)+\left\{G_{\alpha}(\sigma), H_{T}\right\} \approx \\
& \approx \partial_{\tau} G_{\alpha}(\sigma)+\left\{G_{\alpha}(\sigma), H_{0}\right\}+\int d \sigma^{\prime}\left(C_{\alpha \beta}\left(\sigma, \sigma^{\prime}\right) \rho^{\beta}\left(\sigma^{\prime}\right)\right)=0
\end{aligned}
$$

Since $C_{\alpha \beta}$ is nonsingular this equation determines $\rho^{\alpha}$ completely. In other words the gauge symmetry is fixed. Note that after fixing the gauge the original Hamiltonian is strongly zero.

As we have argued above all constraints form the collection of the second class constraints. According to the general theory of constraint systems the proper way how to deal with them is to introduce the Dirac brackets. The Dirac bracket between two phase space functions $F, G$ takes the form

$$
\begin{aligned}
\left\{F(\sigma), G\left(\sigma^{\prime}\right)\right\}_{D} & =\left\{F(\sigma), G\left(\sigma^{\prime}\right)\right\}- \\
& -\int d \sigma^{\prime \prime} d \sigma^{\prime \prime \prime}\left\{F(\sigma), \Phi_{I}\left(\sigma^{\prime}\right)\right\} C^{I J}\left(\sigma^{\prime \prime}, \sigma^{\prime \prime \prime}\right)\left\{\Phi_{J}\left(\sigma^{\prime \prime \prime}\right), G\left(\sigma^{\prime}\right)\right\}
\end{aligned}
$$


where we have introduced following notation

$$
\begin{aligned}
& \Phi_{1}=T_{+}, \quad \Phi_{2}=T_{-}, \quad \Phi_{3}=G_{+}, \quad \Phi_{4}=G_{-}, \\
& \Phi_{5}=\pi_{+}^{\lambda}, \quad \Phi_{6}=G_{+}^{\lambda}, \quad \Phi_{7}=\pi_{-}^{\lambda}, \quad \Phi_{8}=G_{-}^{\lambda}, \\
& \Phi_{9}=\pi_{\xi}, \quad \Phi_{10}=G_{\xi}, \quad C_{I J}\left(\sigma, \sigma^{\prime}\right)=\left\{\Phi_{I}(\sigma), \Phi_{J}\left(\sigma^{\prime}\right)\right\},
\end{aligned}
$$

and where in (3.32) the summation over $I, J$ is understood. Note that $C^{I J}$ is inverse of $C_{I J}$

$$
\int d \sigma^{\prime \prime} C_{I J}\left(\sigma, \sigma^{\prime \prime}\right) C^{J K}\left(\sigma^{\prime \prime}, \sigma^{\prime}\right)=\delta\left(\sigma-\sigma^{\prime}\right) \delta_{I}^{K}
$$

It can be shown the the Dirac brackets of all constraints with any phase space functions are zero. Consequently provided we use Dirac bracket instead of the Poisson bracket, the second class constraint $\Phi_{I}$ can be imposed even before any calculations [34, 35, 36]. We can then explicitly solve these constraints $\Phi_{I}$ leading to the set of coordinates $z_{m}$ that parametrise reduced phase space that is equipped with the bracket structure induced by Dirac bracket. Further, we should find the generator of the time evolution since when all the gauge freedom is fixed the original Hamiltonian strongly vanishes.

The advantage of the gauge fixing is that we study the dynamics of the physical degrees of freedom only. On the other hand it is not completely clear whether the gauge fixing condition allows an existence of an infinite number of conserved charges that are in involution. We postpone the discussion of this problem for future.

On the other hand let us consider an example of the partial gauge fixing that manifestly preserves the integrability. To do this we introduce following gauge fixing condition

$$
G_{+}^{\lambda}=\lambda^{+}-f^{+}, \quad G_{-}^{\lambda}=\lambda^{-}-f^{-}, \quad G_{\xi}=\xi-1,
$$

where $f^{+}, f^{-}$generally depend on $\tau, \sigma$. Note that there is still gauge freedom parametrised by the Lagrange multipliers $\rho^{ \pm}$. Then (3.35) implies following non-zero matrix elements $C$

$$
\begin{aligned}
C_{65}\left(\sigma, \sigma^{\prime}\right) & =-C_{56}\left(\sigma^{\prime}, \sigma\right)=\delta\left(\sigma-\sigma^{\prime}\right), \\
C_{87}\left(\sigma, \sigma^{\prime}\right) & =-C_{87}\left(\sigma^{\prime}, \sigma\right)=\delta\left(\sigma-\sigma^{\prime}\right), \\
C_{9,10}\left(\sigma, \sigma^{\prime}\right) & =-C_{10,9}\left(\sigma^{\prime}, \sigma\right)=\delta\left(\sigma-\sigma^{\prime}\right) .
\end{aligned}
$$

The requirement that the gauge fixing constraints (3.35) have to be preserved during the time evolution determines following values of the Lagrange multipliers $\rho_{ \pm}^{\lambda}, \rho_{\xi}$ :

$$
\rho_{ \pm}^{\lambda}=0, \quad \rho_{\xi}=0 .
$$

Then the partially fixed Hamiltonian takes the form

$$
\begin{aligned}
H & =H_{0}+\int d \sigma\left(\rho^{+} \Phi_{+}+\rho^{-} \Phi_{-}\right)= \\
& =\int d \sigma\left[\left(f^{+}+\rho^{+}\right) T_{+}+\left(f^{-}+\rho^{-}\right) T_{-}\right] .
\end{aligned}
$$


Let us now discuss the consequence of the gauge fixing (3.35) on the form of the current algebra. Since we have not fixed $\rho^{ \pm}$we can again presume that $\rho^{ \pm}$depend on $\tau, \sigma$ only. According to the general analysis we should calculate the Dirac bracket of the current algebra. However it is easy to see from (3.35) that the Dirac bracket of the canonical variables $p_{M}, x^{N}$ coincide with the Poisson bracket. Then the current algebra takes precisely the same form as was determined in the previous section when we replace $\gamma_{\alpha \beta}$ with corresponding combinations of $f^{ \pm}$defined in (3.35). It is also clear that the Poisson bracket of the Lax connection takes the same form as in 3.23). According to the discussion given in the previous section this result ensures an integrability of the theory. In summary principal chiral model with fixed world-sheet metric is integrable.

It is also important to stress that we have not fixed the gauge symmetry generated by $T_{ \pm}$. Then the classical analysis should proceed along the standard treatment of the constraint systems. Explicitly, the constraints $T_{ \pm}$are imposed on the system after performing all calculations. We also demand that the physical observables have vanishing Poisson brackets with the generators of the gauge transformations on the constraint surface. The equivalent requirement on the physical observables is that they commute with the BRST generator corresponding to given gauge symmetries. In fact we have shown in our previous paper [2] that the monodromy matrix is BRST invariant. This result also implies an existence of an infinite number of BRST invariant conserved charges.

\section{Nambu-Gotto form of principal model}

In this section we consider the Nambu-Gotto form of the principal model. It is clear that this form of the string action arises from the Polyakov form of the action through the integration of the world-sheet metric. On the other hand it is not completely clear whether this procedure does not spoil integrability of the theory. For that reason we mean that it is useful to study the integrability of this model as well.

The Nambu-Gotto principal chiral model action takes the form

$$
S=\frac{\sqrt{\lambda}}{2 \pi} \int d \sigma d \tau \mathcal{L}, \quad \mathcal{L}=-\sqrt{-\operatorname{det} \mathbf{A}}
$$

where

$$
\mathbf{A}_{\alpha \beta}=K_{A B} J_{\alpha}^{A} J_{\beta}^{B}, \quad J_{\alpha}=g^{-1} \partial_{\alpha} g=J_{\alpha}^{A} T_{A} .
$$

Let us now consider the variation of the group element $g$ in the form

$$
\delta g=g^{-1} \delta X, \delta X=\delta X^{A} T_{A}
$$

that implies following variation of the current $J$

$$
\delta J_{\alpha}=-\delta X J_{\alpha}+J_{\alpha} \delta X+\partial_{\alpha} \delta X
$$

or explicitly

$$
\delta J_{\alpha}^{A}=J_{\alpha}^{B} f_{B C}^{A} \delta X^{C}+\partial_{\alpha} \delta X^{A}
$$


Then the variation of the action (4.1) under the variation (4.4) takes the form

$$
\begin{aligned}
\frac{2 \pi}{\sqrt{\lambda}} \delta S_{N G} & =\int d \sigma d \tau \frac{1}{2} \delta \mathbf{A}_{\alpha \beta}\left(\mathbf{A}^{-1}\right)^{\beta \alpha} \sqrt{-\operatorname{det} \mathbf{A}}= \\
& =-\delta X^{C}\left(\partial_{\alpha}\left[K_{C B} J_{\beta}^{B}\left(\mathbf{A}^{-1}\right)^{\beta \alpha} \sqrt{-\operatorname{det} \mathbf{A}}\right]-\right. \\
& \left.-f_{C B}^{A} J_{\alpha}^{B} K_{A D} J_{\beta}^{D}\left(\mathbf{A}^{-1}\right)^{\beta \alpha} \sqrt{-\operatorname{det} \mathbf{A}}\right)
\end{aligned}
$$

The last term in the expression above vanishes due to the antisymmetry of $f_{A B}^{C}$ and symmetry of $\left(\mathbf{A}^{-1}\right)^{\alpha \beta}$. If we now demand that the variation of the action vanishes for on-shell configuration we obtain the equation of motion for $J^{A}$ in the form

$$
\partial_{\alpha}\left[J_{\beta}^{A}\left(\mathbf{A}^{-1}\right)^{\beta \alpha} \sqrt{-\operatorname{det} \mathbf{A}}\right]=0 .
$$

Let us now consider following current

$$
\begin{aligned}
& \hat{J}_{\sigma}(\Lambda)=\frac{1}{1-\Lambda^{2}}\left[J_{\sigma}^{A}+\Lambda\left(\mathbf{A}^{-1}\right)^{\tau \alpha} J_{\alpha}^{A} \sqrt{-\operatorname{det} \mathbf{A}}\right] \\
& \hat{J}_{\tau}(\Lambda)=\frac{1}{1-\Lambda^{2}}\left[J_{\tau}^{A}-\Lambda\left(\mathbf{A}^{-1}\right)^{\sigma \alpha} J_{\alpha}^{A} \sqrt{-\operatorname{det} \mathbf{A}}\right]
\end{aligned}
$$

The components of the current given above obey the equation

$$
\begin{aligned}
& \partial_{\tau} \hat{J}_{\sigma}^{A}-\partial_{\sigma} \hat{J}_{\tau}^{A}=\frac{1}{1-\Lambda^{2}}\left[\partial_{\tau} J_{\sigma}^{A}-\partial_{\sigma} J_{\tau}^{A}+\right. \\
+ & \left.\Lambda\left(\partial_{\tau}\left[\left(\mathbf{A}^{-1}\right)^{\tau \alpha} J_{\alpha}^{A} \sqrt{-\operatorname{det} \mathbf{A}}\right]+\partial_{\sigma}\left[\left(\mathbf{A}^{-1}\right)^{\sigma \alpha} J_{\alpha}^{A} \sqrt{-\operatorname{det} \mathbf{A}}\right]\right)\right]= \\
= & -\frac{1}{1-\Lambda^{2}} J_{\tau}^{B} J_{\sigma}^{C} f_{B C}^{A},
\end{aligned}
$$

where we have used the equation of motion (4.6) and the flatness of the current $J_{\alpha}^{A}$. On the other hand let us also calculate

$$
\begin{aligned}
\hat{J}_{\tau}^{B} \hat{J}_{\sigma}^{C} f_{B C}^{A} & =\frac{1}{\left(1-\Lambda^{2}\right)^{2}}\left[J_{\tau}^{B} J_{\sigma}^{C} f_{B C}^{A}+\Lambda J_{\tau}^{B} J_{\sigma}^{C} f_{B C}^{A}\left(\mathbf{A}^{-1}\right)^{\tau \sigma} \sqrt{-\operatorname{det} \mathbf{A}}-\right. \\
& \left.-\Lambda\left(\mathbf{A}^{-1}\right)^{\sigma \tau} J_{\tau}^{B} J_{\sigma}^{C} f_{B C}^{A} \sqrt{-\operatorname{det} \mathbf{A}}-\Lambda^{2}\left(\mathbf{A}^{-1}\right)^{\sigma \alpha} J_{\alpha}^{B}\left(\mathbf{A}^{-1}\right)^{\tau \beta} J_{\beta}^{C} f_{B C}^{A} \sqrt{-\operatorname{det} \mathbf{A}}\right]= \\
& =\frac{1}{\left(1-\Lambda^{2}\right)^{2}}\left(1-\Lambda^{2}\right) J_{\tau}^{B} J_{\sigma}^{C} f_{B C}^{A}
\end{aligned}
$$

using the fact that

$$
-\Lambda^{2}\left(\mathbf{A}^{-1}\right)^{\sigma \alpha} J_{\alpha}^{B}\left(\mathbf{A}^{-1}\right)^{\tau \beta} J_{\beta}^{C} f_{B C}^{A}(\sqrt{-\operatorname{det} \mathbf{A}})^{2}=-\Lambda^{2} J_{\tau}^{B} J_{\sigma}^{C} f_{B C}^{A} .
$$


If we collect (4.8) with (4.9) we obtain an important result

$$
\partial_{\alpha} \hat{J}_{\beta}^{A}-\partial_{\beta} \hat{J}_{\alpha}^{A}+\hat{J}_{\alpha}^{B} \hat{J}_{\beta}^{C} f_{B C}^{A}=0 .
$$

In other words the components of the current (4.7) form the Lax connection and (4.11) implies an integrability of the theory.

Now we proceed to the calculations of the Poisson brackets of the currents. As usual we have to develop the Hamiltonian formalism for the model (4.1). As follows from the form of the action (4.1) the momentum conjugate to $x^{M}$ takes the form

$$
p_{M}=\frac{\delta S}{\delta \partial_{\tau} x^{M}}=-\frac{\sqrt{\lambda}}{2 \pi} G_{M N} \partial_{\alpha} x^{N}\left(\mathbf{A}^{-1}\right)^{\alpha \tau} \sqrt{-\operatorname{det} \mathbf{A}} .
$$

Then it is easy to determine the primary constraint of the theory

$$
\Phi_{0}=\frac{\pi}{\sqrt{\lambda}} p_{M} G^{M N} p_{N}+\frac{\sqrt{\lambda}}{4 \pi} \partial_{\sigma} x^{M} G_{M N} \partial_{\sigma} x^{N}=0 .
$$

At the same time (4.12) implies the second primary constraint

$$
\Phi_{1}=p_{M} \partial_{\sigma} x^{M}=0 .
$$

Since these constraints have exactly the same form as the constraints found in section (2) it is clear that their time evolutions do not imply any secondary constraints. Moreover, the original Hamiltonian density takes the form

$$
\mathcal{H}_{0}=p_{M} \partial_{\tau} x^{M}-\mathcal{L}=0
$$

as we can expect from the fact that the Nambu-Gotto action is diffeomorphism invariant. Then according to the general principles of the dynamics of the constraint systems [34, 35, [36] the total Hamiltonian density takes the form

$$
\mathcal{H}_{T}=\lambda^{0} \Phi_{0}+\lambda^{1} \Phi_{1}
$$

Then it follows that $\partial_{\tau} x^{M}$ is equal to

$$
\partial_{\tau} x^{M}=\left\{x^{M}, H_{T}\right\}=\lambda^{0} \frac{2 \pi}{\sqrt{\lambda}} G^{M N} p_{N}+\lambda^{1} \partial_{\sigma} x^{M} .
$$

Using this result we express the current $J_{\tau}^{A}$ as a function of the canonical variables $p_{M}, x^{M}$

$$
J_{\tau}^{A}=E_{M}^{A} \partial_{\tau} x^{M}=\lambda^{0} \frac{2 \pi}{\sqrt{\lambda}} K^{A B} E_{B}^{N} p_{N}+\lambda^{1} E_{M}^{A} \partial_{\sigma} x^{M} .
$$

This form of the current is the same as the time component of the current given in (3.3) if we perform an identification

$$
\lambda^{0}=-\frac{1}{\sqrt{\tilde{\gamma}} \tilde{\gamma}^{\tau \tau}}, \quad \lambda^{1}=-\frac{\tilde{\gamma}^{\tau \sigma}}{\tilde{\gamma}^{\tau \tau}} .
$$


Without lost of generality we can presume that the Lagrange multipliers $\lambda^{0,1}$ do not depend on the phase space variables and we obtain the Poisson brackets of the current in the form

$$
\begin{aligned}
\left\{J_{\sigma}^{A}(\sigma), J_{\sigma}^{B}\left(\sigma^{\prime}\right)\right\} & =0 \\
\left\{J_{\tau}^{A}(\sigma), J_{\sigma}^{B}\left(\sigma^{\prime}\right)\right\} & =\lambda^{0} \frac{2 \pi}{\sqrt{\lambda}}\left[K^{A B} \partial_{\sigma} \delta\left(\sigma-\sigma^{\prime}\right)+J_{\sigma}^{C} f_{C D}^{A} K^{D B} \delta\left(\sigma-\sigma^{\prime}\right)\right], \\
\left\{J_{\tau}^{A}(\sigma), J_{\tau}^{B}\left(\sigma^{\prime}\right)\right\} & =\lambda_{0} \frac{2 \pi}{\sqrt{\lambda}} J_{\tau}^{C} f_{C D}^{A} K^{D B} \delta\left(\sigma-\sigma^{\prime}\right)-\lambda^{1} \frac{2 \pi}{\sqrt{\lambda}} J_{\sigma}^{C} f_{C D}^{A} K^{D B} \delta\left(\sigma-\sigma^{\prime}\right)+ \\
& +\frac{2 \pi}{\sqrt{\lambda}}\left(\lambda^{0}(\sigma) \lambda^{1}\left(\sigma^{\prime}\right)+\lambda^{0}\left(\sigma^{\prime}\right) \lambda^{1}(\sigma)\right) K^{A B} \partial_{\sigma} \delta\left(\sigma-\sigma^{\prime}\right) .
\end{aligned}
$$

Let us now proceed to the calculation of the Poisson bracket of the spatial component of the Lax connection. Using (4.12) we can write it in the form

$$
\hat{J}_{\sigma}=\frac{1}{1-\Lambda^{2}}\left[J_{\sigma}^{A}-\frac{2 \pi}{\sqrt{\lambda}} \Gamma K^{A B} E_{B}^{M} p_{M}\right]
$$

Then with the help of the formula

$$
\left\{J_{\sigma}^{A}(\sigma), p_{M}\left(\sigma^{\prime}\right)\right\}=E_{M}^{A}(\sigma) \partial_{\sigma} \delta\left(\sigma-\sigma^{\prime}\right)+\partial_{\sigma} x^{N}(\sigma) \partial_{M} E_{N}^{A}(\sigma) \delta\left(\sigma-\sigma^{\prime}\right)
$$

we obtain

$$
\begin{aligned}
& \left\{\hat{J}_{\sigma}^{A}(\Lambda)(\sigma), \hat{J}_{\sigma}^{B}(\Gamma)\left(\sigma^{\prime}\right)\right\}=-\frac{\Gamma+\Lambda}{\left(1-\Gamma^{2}\right)\left(1-\Lambda^{2}\right)} \frac{2 \pi}{\sqrt{\lambda}} K^{A B} \partial_{\sigma} \delta\left(\sigma-\sigma^{\prime}\right)- \\
- & \frac{2 \pi}{\sqrt{\lambda}} \frac{\Gamma+\Lambda}{\left(1-\Gamma^{2}\right)\left(1-\Lambda^{2}\right)} J_{\sigma}^{C} f_{C D}^{A} K^{D B} \delta\left(\sigma-\sigma^{\prime}\right)-\frac{2 \pi}{\sqrt{\lambda}} \frac{\Lambda \Gamma}{\left(1-\Lambda^{2}\right)\left(1-\Gamma^{2}\right)} J_{\tau}^{C} f_{C D}^{A} K^{D B} \delta\left(\sigma-\sigma^{\prime}\right)
\end{aligned}
$$

that again can be rewritten in the more natural form

$$
\begin{aligned}
& \frac{\sqrt{\lambda}}{2 \pi}\left\{\hat{J}_{\sigma}^{A}(\sigma, \Lambda), \hat{J}_{\sigma}^{B}\left(\sigma^{\prime}, \Gamma\right)\right\}=-\frac{K^{A B}(\Gamma+\Lambda)}{\left(1-\Lambda^{2}\right)\left(1-\Gamma^{2}\right)} \partial_{\sigma} \delta\left(\sigma-\sigma^{\prime}\right)- \\
- & \frac{\Gamma^{2}}{\left(1-\Gamma^{2}\right)(\Lambda-\Gamma)} \hat{J}_{\sigma}^{C}(\Lambda) f_{C D}^{A} K^{D B} \delta\left(\sigma-\sigma^{\prime}\right)-\frac{\Lambda^{2}}{\left(1-\Lambda^{2}\right)(\Lambda-\Gamma)} \hat{J}_{\sigma}^{C}(\Gamma) f_{C D}^{A} K^{D B} \delta\left(\sigma-\sigma^{\prime}\right) .
\end{aligned}
$$

Following the careful analysis given in [4] we can now argue that the action (4.1) is integrable and after appropriate regularisation it possesses an infinite number of conserved charges that are in involution.

\section{Conclusion}

This short note was devoted to the calculation of the algebra of the current for principal chiral model coupled to two dimensional gravity. We have determined the algebra of 
currents that depend on the world-sheet metric and on the gauge parameters. Then we have calculated the Poisson bracket of the spatial component of the Lax connection and we have shown that this result does not depend on the metric and gauge parameters. This fact implies an existence of infinite number of gauge independent conserved charges that are in involution. We have also shown that the same conclusions are valid for the Nambu-Gotto form of the action.

The extension of this work is as follows. We mean that it is very interesting problem to study the integrability of the bosonic string on $A d S_{5} \times S^{5}$ in the uniform light-cone gauge [29, 30]. Even if it was shown in [14] that it is possible to define the Lax connection for the gauge fixed form of the action as well, the calculation of the Poisson bracket of the spatial component of the Lax connection has not been performed yet. We think that it would be very useful to know whether the gauge fixed form of the action possesses infinite number of the integrals of motions that are in involution. We hope to return to this problem in future.

\section{Acknowledgements}

I would like to thank J. Govaerts for very interesting and stimulating correspondence. This work was supported in part by the Czech Ministry of Education under Contract No. MSM 0021622409, by INFN, by the MIUR-COFIN contract 2003-023852 and, by the EU contracts MRTN-CT-2004-503369 and MRTN-CT-2004-512194, by the INTAS contract 03-516346 and by the NATO grant PST.CLG.978785.

\section{References}

[1] I. Bena, J. Polchinski and R. Roiban, "Hidden symmetries of the AdS(5) x $S^{* * 5}$ superstring," Phys. Rev. D 69 (2004) 046002 [arXiv:hep-th/0305116].

[2] J. Kluson, "BRST invariance of non-local charges and monodromy matrix of bosonic string on $\operatorname{AdS}(5) \times S^{* *}$," arXiv:hep-th/0701013.

[3] P. A. Grassi and J. Kluson, "Pure spinor strings in TsT deformed background," arXiv:hep-th/0611151.

[4] N. Dorey and B. Vicedo, "A symplectic structure for string theory on integrable backgrounds," arXiv:hep-th/0606287.

[5] N. Gromov, V. Kazakov, K. Sakai and P. Vieira, "Strings as multi-particle states of quantum sigma-models," Nucl. Phys. B 764 (2007) 15 [arXiv:hep-th/0603043].

[6] L. F. Alday, G. Arutyunov and S. Frolov, "Green-Schwarz strings in TsT-transformed backgrounds,” JHEP 0606 (2006) 018 [arXiv:hep-th/0512253].

[7] A. Das, A. Melikyan and M. Sato, "The algebra of flat currents for the string on AdS(5) $x$ $S^{* * 5}$ in the light-cone gauge," JHEP 0511 (2005) 015 [arXiv:hep-th/0508183].

[8] L. F. Alday, G. Arutyunov and S. Frolov, "New integrable system of 2dim fermions from strings on AdS(5) x $S^{* * 5}$," JHEP 0601 (2006) 078 [arXiv:hep-th/0508140].

[9] G. Arutyunov and M. Zamaklar, "Linking Baecklund and monodromy charges for strings on $A d S(5) x S^{* *} 5$," JHEP 0507 (2005) 026 [arXiv:hep-th/0504144]. 
[10] S. Frolov, "Lax pair for strings in Lunin-Maldacena background," JHEP 0505 (2005) 069 [arXiv:hep-th/0503201].

[11] B. Chen, Y. L. He, P. Zhang and X. C. Song, "Flat currents of the Green-Schwarz superstrings in $\operatorname{AdS}(5) x S^{* * 1}$ and $A d S(3) x S^{* * 3}$ backgrounds," Phys. Rev. D 71 (2005) 086007 [arXiv:hep-th/0503089].

[12] L. F. Alday, G. Arutyunov and A. A. Tseytlin, "On integrability of classical superstrings in $A d S(5) x S^{* *} 5$," JHEP 0507 (2005) 002 [arXiv:hep-th/0502240].

[13] A. Das, J. Maharana, A. Melikyan and M. Sato, "The algebra of transition matrices for the $\operatorname{AdS(5)} x S^{* *} 5$ superstring," JHEP 0412 (2004) 055 [arXiv:hep-th/0411200].

[14] G. Arutyunov and S. Frolov, "Integrable Hamiltonian for classical strings on $\operatorname{AdS}(5) x S^{* * 5}$," JHEP 0502 (2005) 059 [arXiv:hep-th/0411089].

[15] N. Beisert, V. A. Kazakov and K. Sakai, "Algebraic curve for the SO(6) sector of AdS/CFT," Commun. Math. Phys. 263 (2006) 611 [arXiv:hep-th/0410253].

[16] V. A. Kazakov and K. Zarembo, "Classical / quantum integrability in non-compact sector of AdS/CFT," JHEP 0410 (2004) 060 [arXiv:hep-th/0410105].

[17] N. Berkovits, "BRST cohomology and nonlocal conserved charges," JHEP 0502 (2005) 060 [arXiv:hep-th/0409159].

[18] M. Hatsuda and K. Yoshida, "Classical integrability and super Yangian of superstring on $A d S(5) x S^{* *} 5$," Adv. Theor. Math. Phys. 9 (2005) 703 [arXiv:hep-th/0407044].

[19] V. A. Kazakov, A. Marshakov, J. A. Minahan and K. Zarembo, "Classical / quantum integrability in AdS/CFT,” JHEP 0405 (2004) 024 [arXiv:hep-th/0402207].

[20] L. F. Alday, "Non-local charges on AdS(5) x $S^{* * 5}$ and pp-waves," JHEP 0312 (2003) 033 [arXiv:hep-th/0310146].

[21] B. C. Vallilo, "Flat currents in the classical AdS(5) $x S^{* *} 5$ pure spinor superstring," JHEP 0403 (2004) 037 [arXiv:hep-th/0307018].

[22] L. D. Faddeev and L. A. Takhtajan, "HAMILTONIAN METHODS IN THE THEORY OF SOLITONS,"

[23] H. J. de Vega, H. Eichenherr and J. M. Maillet, "Classical And Quantum Algebras Of Nonlocal Charges In Sigma Models," Commun. Math. Phys. 92 (1984) 507.

[24] A. G. Izergin and V. E. Korepin, "The Inverse Scattering Method Approach To The Quantum Shabat-Mikhailov Model," Commun. Math. Phys. 79, 303 (1981).

[25] D. Korotkin and H. Samtleben, "Yangian symmetry in integrable quantum gravity," Nucl. Phys. B 527 (1998) 657 [arXiv:hep-th/9710210].

[26] J. M. Maillet, "New Integrable Canonical Structures In Two-Dimensional Models," Nucl. Phys. B 269 (1986) 54.

[27] J. M. Maillet, "Hamiltonian Structures For Integrable Classical Theories From Graded Kac-Moody Algebras," Phys. Lett. B 167 (1986) 401.

[28] N. Dorey and B. Vicedo, "On the dynamics of finite-gap solutions in classical string theory," JHEP 0607 (2006) 014 [arXiv:hep-th/0601194]. 
[29] G. Arutyunov, S. Frolov and M. Zamaklar, "Finite-size effects from giant magnons," arXiv:hep-th/0606126.

[30] G. Arutyunov and S. Frolov, "Uniform light-cone gauge for strings in AdS(5) x $S^{* *} 5$ : Solving su(1-1) sector," JHEP 0601 (2006) 055 [arXiv:hep-th/0510208].

[31] T. Klose, T. McLoughlin, R. Roiban and K. Zarembo, "Worldsheet scattering in AdS(5) $x$ $S^{* *} 5$," arXiv:hep-th/0611169.

[32] D. Astolfi, V. Forini, G. Grignani and G. W. Semenoff, "Gauge invariant finite size spectrum of the giant magnon," arXiv:hep-th/0702043.

[33] J. M. Evans, M. Hassan, N. J. MacKay and A. J. Mountain, "Local conserved charges in principal chiral models," Nucl. Phys. B 561 (1999) 385 [arXiv:hep-th/9902008].

[34] M. Henneaux and C. Teitelboim, "Quantization of gauge systems," Princeton, USA: Univ. $\operatorname{Pr.~(1992)~} 520 \mathrm{p}$

[35] J. Govaerts, "Hamiltonian Quantization And Constrained Dynamics," Leuven, Belgium: Univ. Pr. (1991) 371 p. (Leuven notes in mathematical and theoretical physics, B4)

[36] J. Govaerts, "The quantum geometer's universe: Particles, interactions and topology," arXiv:hep-th/0207276. 